\title{
Diagnóstico clínico y aspecto psicosocial de trastornos temporomandibulares según el índice CDI/TTM en estudiantes de odontología
}

Clinical diagnosis and psychosocial aspect of temporomandibular disorders according to the CDI / TTM index in dental students

Cynthia Rojas-Martinez ${ }^{1, a}$, Felipe Enrique Lozano-Castro ${ }^{1, a, b, c}$

\section{RESUMEN}

Objetivo: El presente trabajo determinó la relación entre el diagnóstico y el aspecto psicosocial del Trastorno Temporomandibular (TTM) según el eje I y II del Índice de Criterios Diagnósticos para la Investigación de Trastornos Temporomandibulares (CDI/TTM), en estudiantes de Facultad de Odontología de la Universidad Nacional Mayor de San Marcos, Lima (Perú) durante el año 2013. Material y métodos: La muestra estuvo conformada por 76 estudiantes de 18 a 30 años de edad con diagnóstico de TTM. Previamente se realizó un estudio piloto bajo ciertos criterios de inclusión y exclusión. Los datos fueron analizados mediante estadística descriptiva; la hipótesis fue contrastada con la prueba estadística chi-cuadrado y la correlación de Spearman, aplicando el paquete estadístico SPSS 21.0. Resultados: Los trastornos musculares se presentaron en 11,84\%; desplazamiento del disco articular en 55,3\% y otras condiciones articulares en 32,9\%. Para trastornos musculares y desplazamiento del disco articular, las mujeres presentaron mayor prevalencia siendo estadísticamente significativo $(\mathrm{p}=0.006)$. El desplazamiento del disco articular con reducción fue más frecuente en la articulación derecha con $31,6 \%$ que en la izquierda con $22,4 \%$. Con respecto al eje II; el tipo de dolor crónico que presentó mayor frecuencia fue de grado I con $28,9 \%$, la depresión severa fue $84,2 \%$ y la somatización severa fue $77,6 \%$. Se encontró relación directa entre trastornos musculares y grado de dolor crónico (Correlación Spearman Rho $=$ 0.472; $\mathrm{p}=0.000)$. Conclusiones: Se encontró una relación directa entre trastornos musculares y el grado de dolor crónico. El desplazamiento del disco articular con reducción fue más frecuente para los TTM con predominio en mujeres.

PALABRAS CLAVE: Trastorno de la articulación temporomandibular, dolor crónico, depresión. (DeCS, Bireme)

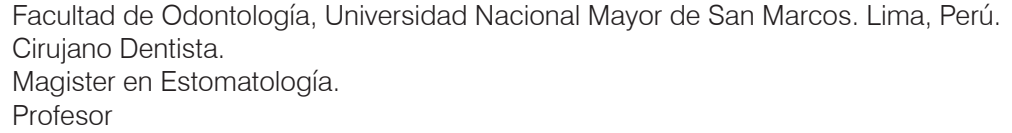




\section{SUMMARY}

Objective: This study determined the relationship between diagnosis and psychosocial aspect of TMD as axis I and II of the Research Diagnostic Criteria for Temporomandibular Disorders Index (RDC/TMD) in students of the school of Dentistry at Universidad Nacional Mayor de San Marcos, Lima (Perú) at 2013. Methods: The sample consisted of 76 students from 18 to 30 years old with diagnostic of TMD. Previously, a pilot study was applied with inclusion and exclusion criteria. Data were analyzed using descriptive statistics, the hypothesis was tested with the chi-square and Spearman correlation tests statistics using the SPSS 21.0. statistical package. Results: Muscle disorders occurred in $11.84 \%$, articular disc displacement in $55.3 \%$ and other joint conditions in $32.9 \%$. For Muscle disorders and articular disc displacement women showed a higher prevalence, which was statistically significant $(p=0.006)$. The articular disc displacement with reduction was the most frequent diagnosis for the right joint with $31.6 \%$ and for the left $22.4 \%$. In relation to axis II; the most frequent grade for chronic pain was Grade I with $28.9 \%$, severe depression was $84.2 \%$ y severe somatization was $77.6 \%$. There was a direct relationship between muscle disorders and chronic pain grade (Spearman correlation test Rho $=0.472$; $p=0.000)$. Conclusions: A direct relation was present between muscle disorders and chronic pain condition. Disc displacement with reduction was the most frequent condition between TMD, predominantly in women.

\section{KEYWORDS: Temporomandibular joint disorder, chronic pain, depression. (MeSH, NLM)}

\section{INTRODUCCIÓN}

La Asociación Dental Americana (ADA) adoptó el término de Trastornos Temporomandibulares (TTM) para referirse a un grupo heterogéneo de condiciones clínicas de la articulación temporomandibular, músculos de la masticación y estructuras anatómicas adyacentes (1).

La etiología de los TTM es multifactorial (2), no sólo involucra aspectos fisiológicos; sino también, también aspectos psicológicos y sociales (3). Hasta el punto que las manifestaciones de dolor influyen en la calidad de vida y discapacidad en las actividades diarias (4).

Dworkin (5) considera a los TTM como trastornos psicofisiológicos y a los factores psicosociales como estrés, ansiedad y depresión como predisponentes en la perpetuación de síntomas severos de TTM.

Numerosas investigaciones $(3,6,7)$ han revelado la prevalencia variable de TTM que va de $1 \%$ a $93 \%$ debido a la falta de métodos de examen estandarizados válidos, confiables y homogeneidad en los criterios diagnósticos $(8,9)$.

En el Perú, se han reportado prevalencias del $31,8 \%$ al $91,42 \%(10,11)$. Del alto porcentaje, sólo el $2 \%$ al $7 \%$ de pacientes afectados buscan tratamiento $(10,6,11)$.
Aunque los TTM se pueden presentar a cualquier edad, la mayor población comúnmente son adultos jóvenes (12 - 14).

La relación entre género y TTM también ha sido ampliamente discutida en la literatura. (9 - 13) Los TTM son considerados 10 veces más frecuente en mujeres, principalmente en edad reproductiva, entre los 20 y 40 años $(7,13,15,16)$.

Para la evaluación de los TTM, se han propuesto diversos índices, tales como los índices de: Organización Mundial de la Salud (1962) (6), KroghPaulsen (1969) (6,17), Martí Helkimo (1971) (18), Maglione (1976) (19), Fricton y Schiffman (1986) (6, 16).

Los índices antes mencionados sólo dan referencia de la presencia y severidad de TTM, más no el diagnóstico, ni la clasificación de los mismos. Tampoco incluyen la valoración de los aspectos psicosociales, importante en el estudio de TTM.

Ante esta problemática, Dworkin y LeResche (16) en 1992, elaboraron el Índice de Criterios Diagnósticos para la Investigación de Trastornos Temporomandibulares (CDI/TTM). Éste consta de dos ejes: a) el eje I, se basa en el cuestionario anamnésico y examen clínico; y b) el eje II, abarca 
el estado psicológico, la discapacidad y el dolor relacionado con los TTM. Éste índice presenta criterios estandarizados para la investigación de TTM, maximiza la confiabilidad y minimiza la variabilidad de los métodos de examinación (16).

El índice de CDI/TTM viene siendo utilizado en la Facultad de Odontología de la Universidad Nacional Mayor de San Marcos (FO-UNMSM) desde el año 2011, dándose mayor énfasis al eje I.

Estudios realizados en nuestro país no han evaluado ambos ejes; motivo por el cual, se consideró importante determinar si en la población elegida existía relación entre ambos ejes.

El objetivo del estudio fue determinar la relación entre el diagnóstico y aspecto psicosocial de TTM según el índice CDI/TTM en estudiantes adultos jóvenes de la FO-UNMSM en el periodo comprendido entre agosto - setiembre del año 2013.

\section{MATERIAL Y MÉTODOS}

Se realizó un estudio correlacional de corte transversal en una muestra no probabilística por conveniencia. La población fue de 201 estudiantes adultos jóvenes de 18 a 30 años de edad, que cursaron el 6to, 8vo y $10 \mathrm{mo}$ semestre académico de la FOUNMSM en el periodo agosto a setiembre del año 2013, con diagnóstico de TTM según el índice CDI/TTM de manera intencional. Se excluyeron a aquellos con: enfermedades sistémicas o neurológicas, tratamiento ortodóntico, disfunción temporomandibular, rehabilitación oral o tratamiento quirúrgico; y a aquellos que el día del examen presentaron otalgia. Se tomó una muestra intencional de 76 estudiantes. El estudio se realizó en las instalaciones de la FOUNMSM mediante un cuestionario anamnésico y examen clínico. Se confeccionó un instrumento para la validación del índice CDI/TTM por juicio de expertos; para ello se contó con 3 cirujanos dentistas especialistas en el área de Rehabilitación Oral, determinándose que el instrumento era válido para aplicarlo. Se realizó un estudio piloto en el 10\% de la muestra $(n=8)$ y se encontró excelente confiabilidad (Alfa de Cronbach $=0,717$ ) según Herrera (20). Los sujetos que participaron en la investigación previamente firmaron un consentimiento informado. Para el examen clínico, se utilizó el calibrador digital marca KAMASA ${ }^{\circledR}$ y se registraron las mediciones en $\mathrm{mm}$. El cuestionario anamnésico y el examen clínico se realizó en función a la guía de procedimientos de CDI/TTM.

Los resultados fueron analizados mediante estadística descriptiva obteniendo porcentajes y frecuencias. La hipótesis fue contrastada con la prueba estadística chi-cuadrado y correlación de Spearman para las variables ordinales, utilizándose el paquete estadístico SPSS 21.0. Se utilizó el programa Microsoft Excel 2010 para realizar gráficos.

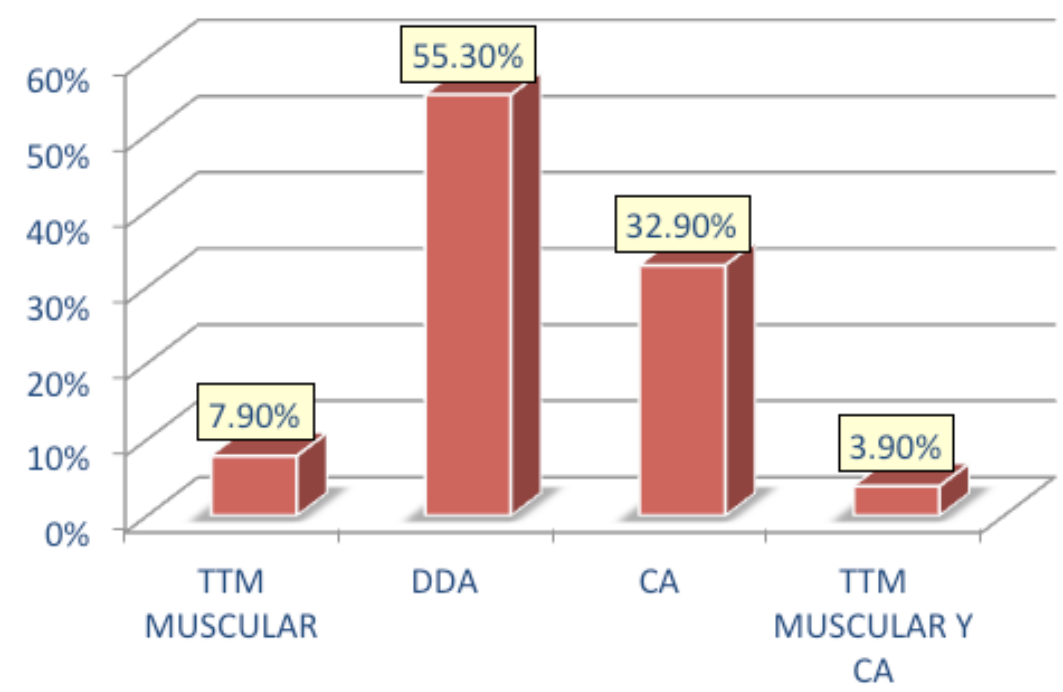

Gráfico 1. Frecuencia de trastornos temporomandibulares según el índice CDI/TTM en estudiantes de odontología, FO-UNMSM. 


\section{RESULTADOS}

Eje I:

De los 76 estudiantes adultos jóvenes seleccionados, el 96,1\% presentó un sólo diagnóstico de trastorno temporomandibular, siendo más frecuente el desplazamiento del disco articular (55,3\%). Por otra parte, el diagnóstico doble (trastorno muscular y condición articular) se presentó en el 3,9\%. (Gráfico $1)$.

\section{Frecuencia de TTM según género}

La frecuencia de trastornos temporomandibulares fue $56,6 \%$ en mujeres y $43,4 \%$ en hombres. Las mujeres presentaron mayor prevalencia en la mayoría de los diagnósticos de TTM. La diferencia fue estadísticamente significativa $\left(\mathrm{x}^{2}=12,454 ; \mathrm{p}<0,05\right)$, a excepción de condiciones articulares (Tabla 1).

\section{Frecuencia de trastornos musculares}

Los trastornos musculares se presentaron en $11,8 \%$ de la muestra. El dolor miofascial se presentó en $6,6 \%$ (Gráfico 2). No se observó diferencia estadísticamente significativa entre mujeres $(14 \%) \mathrm{y}$ hombres $(9,1 \%)$.

\section{Frecuencia de desplazamiento del disco articular}

El desplazamiento del disco articular se presentó en el 55,3\% de la muestra. El desplazamiento del disco articular con reducción fue el diagnóstico más frecuente en la articulación derecha $(31,6 \%)$ que en la articulación izquierda (22,4\%) (Gráfico 3).
Se observó diferencia estadísticamente significativa en los resultados entre mujeres $(69,8 \%)$ y hombres $(36,4 \%)\left(x^{2}=8,717 ; \mathrm{p}<0,05\right)$.

\section{Frecuencia de condiciones articulares}

Las condiciones articulares se presentaron en el $36,8 \%$ de los individuos examinados. El 14,5\% de la muestra presentó diagnóstico de condiciones articulares del lado derecho e izquierdo. La osteoartrosis de la articulación derecha $(23,7 \%)$ e izquierda $(18,4 \%)$ fueron las condiciones más frecuentes (Gráfico 4). Se observó diferencia estadísticamente significativa entre los resultados de mujeres $20,9 \%$ y hombres $57,5 \%$. Con $p<0,05$.

\section{Eje II:}

Frecuencia de aspectos psicosociales según género

La frecuencia de aspectos psicosociales fue de $100 \%$ en mujeres y $90,9 \%$ en hombres. No se encontró diferencia estadísticamente significativa entre ambos (Tabla 2).

\section{Frecuencia de grado de dolor crónico}

Algún grado de dolor crónico se presentó en el $43,4 \%$ del total de la muestra $(n=33)$, siendo el grado I el más frecuente con 28,9\% (Gráfico 5). No se encontró diferencia estadísticamente significativa entre mujeres $(44,2 \%)$ y hombres $(42,4 \%)$.

Tabla 1. Frecuencia de trastornos temporomandibulares según género en estudiantes de odontología, FO-UNMSM.

\begin{tabular}{|c|c|c|c|c|c|c|}
\hline \multirow{3}{*}{$\begin{array}{c}\text { TRASTORNOS } \\
\text { TEMPOROMANDIBULARES }\end{array}$} & \multicolumn{4}{|c|}{ GENERO } & \multirow{2}{*}{\multicolumn{2}{|c|}{ Total }} \\
\hline & \multicolumn{2}{|c|}{ Masculino } & \multicolumn{2}{|c|}{ Femenino } & & \\
\hline & $\mathbf{n}$ & $\%$ & $\mathbf{n}$ & $\%$ & $\mathbf{n}$ & $\%$ \\
\hline Trastorno muscular & 2 & $2.6 \%$ & 4 & $5.3 \%$ & 6 & $7.9 \%$ \\
\hline $\begin{array}{l}\text { Desplazamiento del disco } \\
\text { articular }\end{array}$ & 12 & $15.8 \%$ & 30 & $39.5 \%$ & 42 & $55.3 \%$ \\
\hline Condiciones articulares & 18 & $23.7 \%$ & 7 & $9.2 \%$ & 25 & $32.9 \%$ \\
\hline $\begin{array}{c}\text { Trastorno muscular y condiciones } \\
\text { articulares }\end{array}$ & 1 & $1.3 \%$ & 2 & $2.6 \%$ & 3 & $3.9 \%$ \\
\hline Total & 33 & $43.4 \%$ & 43 & $56.6 \%$ & 76 & $100 \%$ \\
\hline
\end{tabular}

Prueba $\mathrm{x} 2=12.454 \mathrm{p}=0.006, \mathrm{p}<0.05$ 


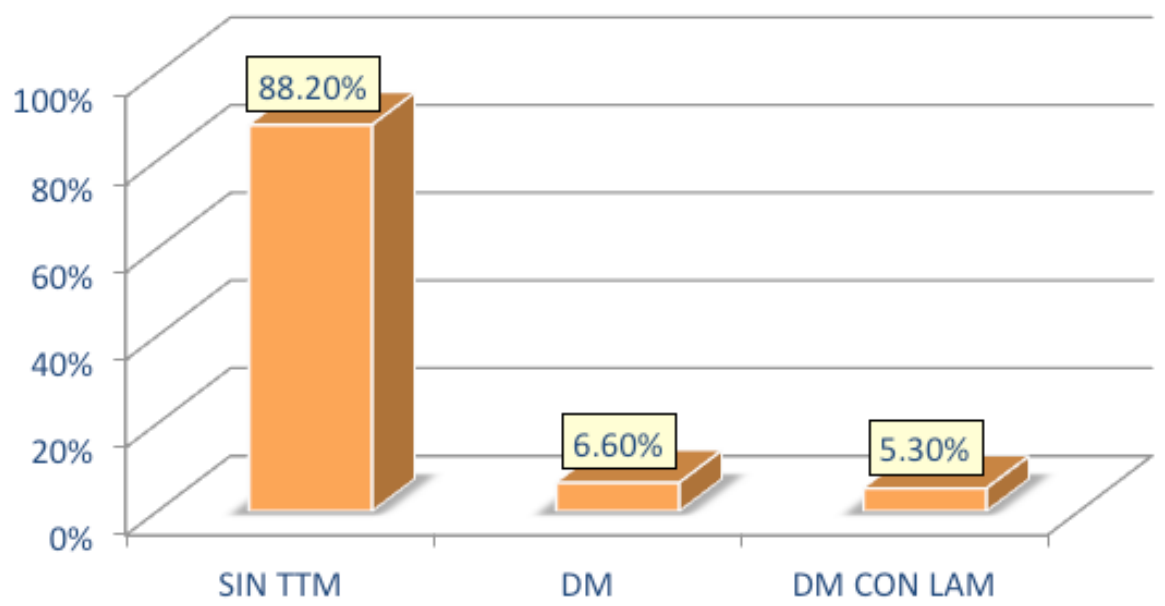

Gráfico 2. Frecuencia de trastornos musculares según el índice CDI/TTM en estudiantes de odontología, FO-UNMSM.

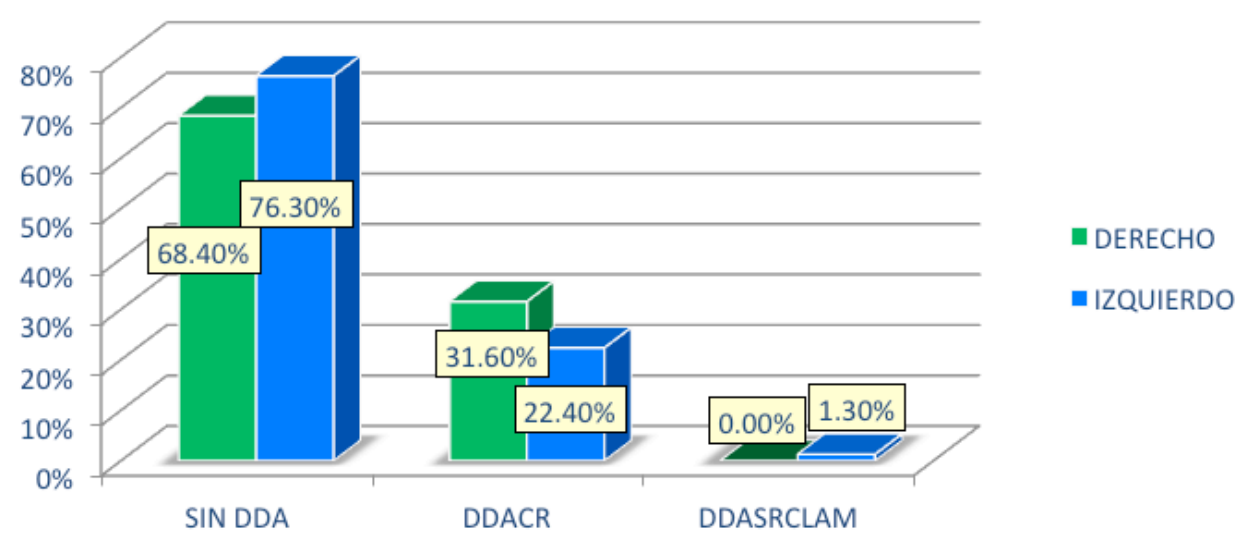

Gráfico 3. Frecuencia de desplazamiento del disco articular según el índice CDI/TTM en estudiantes de odontología, FO-UNMSM.

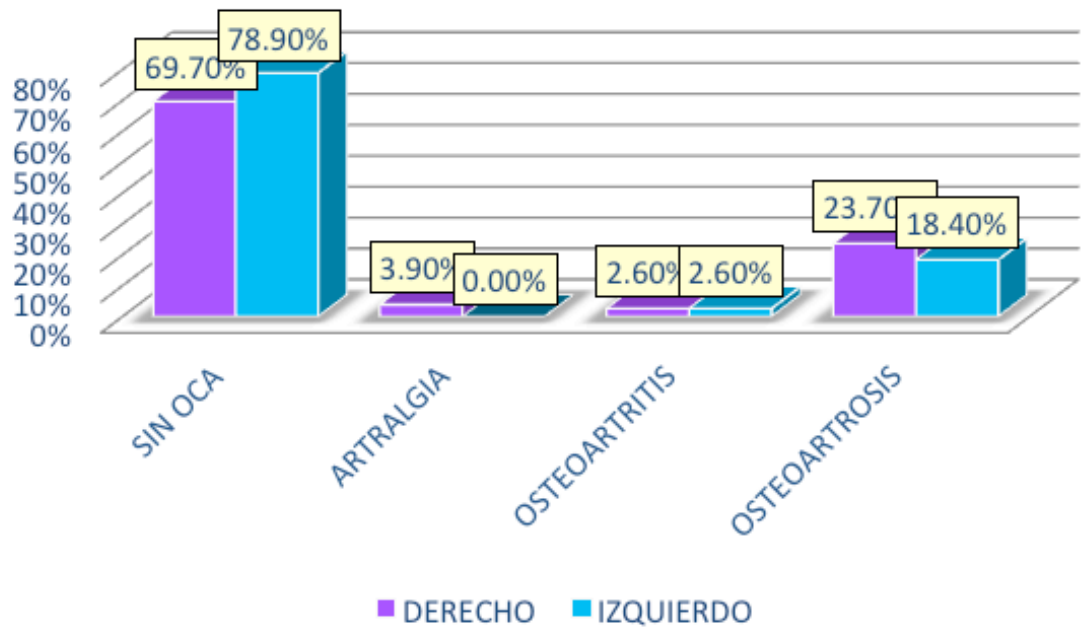

Gráfico 4. Frecuencia de condiciones articulares, según el índice CDI/TTM en estudiantes de odontología, FO-UNMSM. 
Tabla 2. Frecuencia de aspectos psicosociales según género en estudiantes de odontología, FO-UNMSM.

\begin{tabular}{|c|c|c|c|c|c|c|}
\hline \multirow{3}{*}{ Aspectos Psicosociales } & \multicolumn{4}{|c|}{ Género } & \multicolumn{2}{|c|}{ Total } \\
\hline & \multicolumn{2}{|c|}{ Masculino } & \multicolumn{2}{|c|}{ Femenino } & \multirow[b]{2}{*}{$\mathbf{n}$} & \multirow[b]{2}{*}{$\%$} \\
\hline & $\mathbf{n}$ & $\%$ & $\mathbf{n}$ & $\%$ & & \\
\hline Ninguno & 3 & $9.1 \%$ & 0 & $0 \%$ & 3 & $3.9 \%$ \\
\hline Depresión & 1 & $3 \%$ & 1 & $2.3 \%$ & 2 & $2.6 \%$ \\
\hline $\begin{array}{c}\text { Grado de dolor crónico y de- } \\
\text { presión }\end{array}$ & 1 & $3 \%$ & 0 & $0 \%$ & 1 & $1.3 \%$ \\
\hline $\begin{array}{c}\text { Grado de dolor crónico y soma- } \\
\text { tización }\end{array}$ & 2 & $6.1 \%$ & 1 & $2.3 \%$ & 3 & $3.9 \%$ \\
\hline Depresión y somatización & 15 & $45.5 \%$ & 23 & $53.5 \%$ & 38 & $50 \%$ \\
\hline $\begin{array}{l}\text { Grado de dolor crónico, depre- } \\
\text { sión y somatización }\end{array}$ & 11 & $33.3 \%$ & 18 & $41.9 \%$ & 29 & $38.2 \%$ \\
\hline Total & 33 & $100 \%$ & 43 & $100 \%$ & 76 & $100 \%$ \\
\hline
\end{tabular}

Prueba $\mathrm{x}^{2}=6.504 \mathrm{p}=0.260$

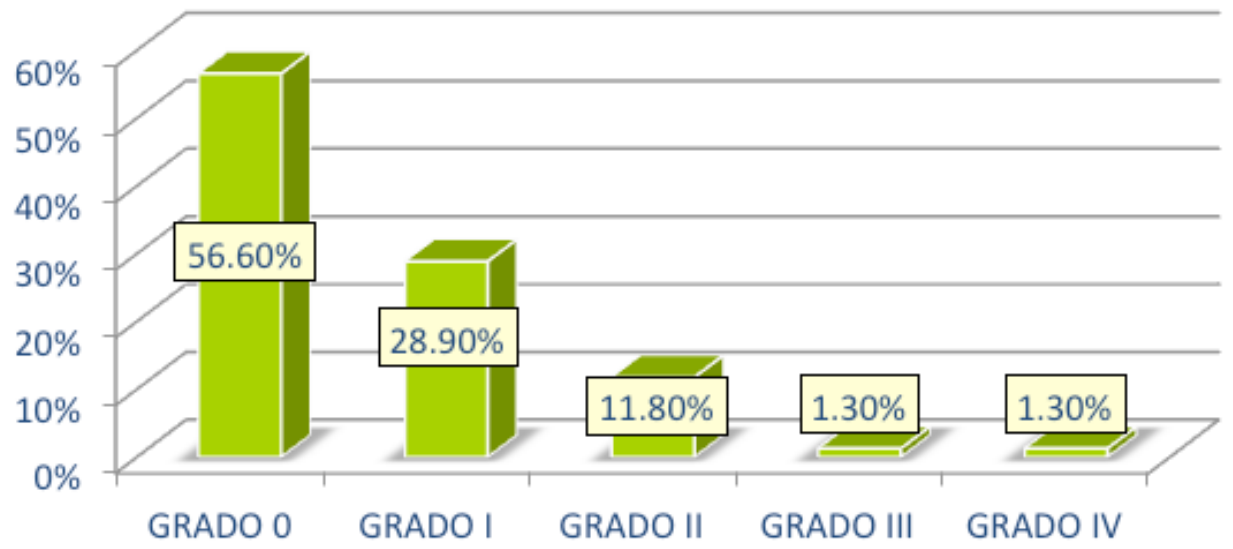

Gráfico 5. Frecuencia de grado de dolor crónico según el índice CDI/TTM en estudiantes de odontología, FO-UNMSM.

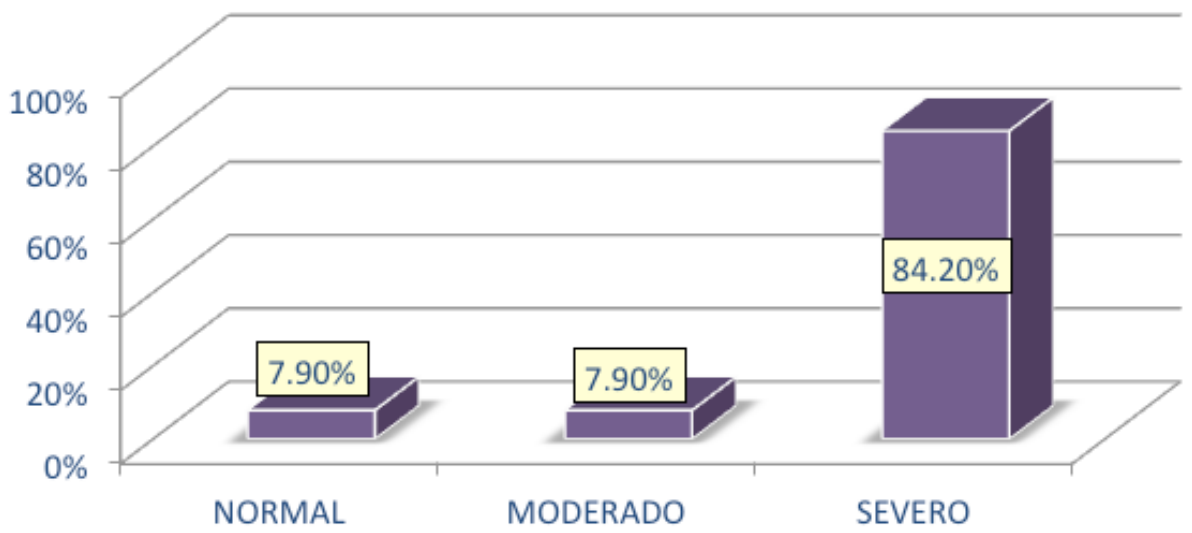

Gráfico 6. Frecuencia de nivel de depresión según el índice CDI/TTM en estudiantes de odontología, FO-UNMSM. 


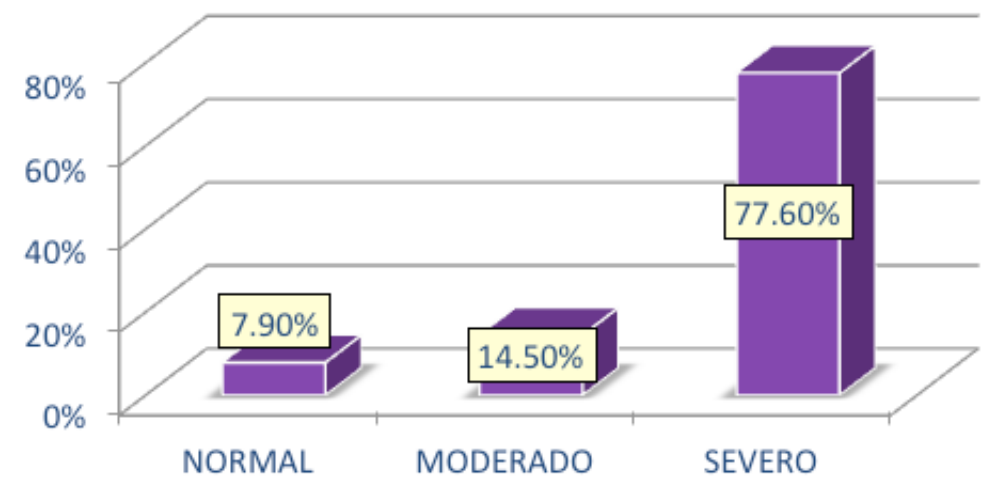

Gráfico 7. Frecuencia del nivel de somatización según el índice CDI/TTM en estudiantes de odontología, FO-UNMSM.

Tabla 3. Relación entre trastorno muscular y grado de dolor crónico según el índice CDI/TTM en estudiantes de odontología, FO-UNMSM.

\begin{tabular}{|c|c|c|c|c|c|c|c|c|c|c|c|c|}
\hline \multirow{3}{*}{$\begin{array}{l}\text { Trastorno } \\
\text { muscular }\end{array}$} & \multicolumn{11}{|c|}{ Grado de dolor Crónico } & \multirow[b]{2}{*}{ Total } \\
\hline & \multicolumn{2}{|c|}{ Grado 0} & \multicolumn{2}{|c|}{ Grado I } & \multicolumn{2}{|c|}{ Grado II } & \multicolumn{2}{|c|}{ Grado III } & \multicolumn{2}{|c|}{ Grado IV } & & \\
\hline & $\mathrm{n}$ & $\%$ & $\mathrm{n}$ & $\%$ & $\mathbf{n}$ & $\%$ & $\mathrm{n}$ & $\%$ & $\mathbf{n}$ & $\%$ & $\mathbf{n}$ & $\%$ \\
\hline $\begin{array}{l}\text { Sin trastorno } \\
\text { muscular }\end{array}$ & 43 & $56.6 \%$ & 18 & $23.7 \%$ & 5 & $6.6 \%$ & 1 & $1.3 \%$ & 0 & $0 \%$ & 67 & $88.2 \%$ \\
\hline Dolor miofascial & 0 & $0 \%$ & 2 & $2.6 \%$ & 2 & $2.6 \%$ & 0 & $0 \%$ & 1 & $1.3 \%$ & 5 & $6.6 \%$ \\
\hline $\begin{array}{l}\text { Dolor miofascial } \\
\text { con limitación } \\
\text { de apertura } \\
\text { mandibular }\end{array}$ & 0 & $0 \%$ & 2 & $2.6 \%$ & 2 & $2.6 \%$ & 0 & $0 \%$ & 0 & $0 \%$ & 4 & $5.3 \%$ \\
\hline Total & 43 & $56.6 \%$ & 22 & $28.9 \%$ & 9 & $11.8 \%$ & 1 & $1.3 \%$ & 1 & $1.3 \%$ & 76 & $100 \%$ \\
\hline
\end{tabular}

Prueba $\mathrm{x} 2=30.385 \mathrm{p}=0.000, \mathrm{p}<0.05$

Prueba de Correlación Spearman $\mathrm{p}=0.000, \mathrm{p}<0.05 \mathrm{Rho}=0.472$

\section{Frecuencia de nivel de depresión}

La depresión se presentó en el 92,1\% (Gráfico 6). El nivel más frecuente fue el severo $(84,2 \%)$. No se observó diferencia significativa entre mujeres $(91,4 \%)$ y hombres $(84,9 \%)$.

\section{Frecuencia de nivel de somatización}

La somatización se encontró en el 92,1\%. El nivel más frecuente fue el severo con 77,6\% (Gráfico 7). No se observó diferencia estadísticamente significativa entre mujeres $(97,6 \%)$ y hombres $(84,9 \%)$.

\section{Relación entre diagnóstico y aspecto psicosocial de TTM}

Sólo se observó correlación positiva entre trastornos musculares y el grado de dolor crónico $($ Rho $=0,472 ; \mathrm{p}<0,05)$, lo que indica que a medida que aumentó la severidad de los trastornos musculares existió mayor grado de dolor crónico (Tabla 3).

\section{DISCUSIÓN}

Diversas investigaciones se han realizado sobre TTM utilizando el índice CDI/TTM $(6,8,16)$, la mayoría de ellas hace énfasis sólo en el eje I (16), muy pocos trabajos publicados son los que han buscado una relación entre ambos ejes $(3,4,21,23)$.

En el presente estudio se encontró que la prevalencia de TTM según género fue mayor en mujeres, presentando diferencia estadísticamente significativa, lo cual es consistente con estudios realizados $(21,22,9,19,6)$. Esto se podría explicar porque las mujeres están sometidas a niveles de 
estrés psicofisiológico más elevados y presentan diferencias fisiológicas como variaciones hormonales como el estrógeno y la progesterona que tienen efecto metabólico en las células del cartílago y del hueso y aumentan la vigilancia frente al dolor (25-26).

Se pudo observar que el CDI/TTM no sólo nos da un diagnóstico, hubo un pequeño porcentaje $(3,9 \%)$ que presentó diagnóstico doble (trastornos musculares + condiciones articulares), lo cual confirma la importancia clínica de describir la prevalencia de trastornos combinados. El estudio de Manfredini y col. (8) muestra que el $64,3 \%$ de la muestra estudiada recibieron diagnósticos múltiples, porcentaje mucho mayor a lo encontrado en el presente estudio, posiblemente porque su muestra fue mayor (8).

En cuanto a los trastornos musculares (solos o combinados) se encontró un $11,8 \%$, porcentaje mucho menor a lo reportado por Espinosa y col. (23). Otros resultados han sido encontrados en los que ésta alteración varía entre $31 \%$ reportado en pacientes asiáticos (2) y 76\% en suecos (3) y americanos (6). Con respecto al desplazamiento del disco articular, éste se encontró en el 55,3\% de la muestra; porcentaje similar al encontrado por Manfredini y col. (8) de $57,3 \%(8)$.

El desplazamiento del disco articular con reducción fue común en todos los diagnósticos del eje I con frecuencias de $31,6 \%$ y $22,4 \%$ para la articulación derecha e izquierda respectivamente. Estos datos concuerdan con los hallazgos de la literatura $(21,22$ y 23) que informaron una prevalencia que oscila entre $10 \%$ y $34 \%$ para cada articulación.

Las condiciones articulares se evidenciaron en el $36,8 \%$ de la muestra, siendo la osteoartrosis el diagnóstico frecuente con $23,7 \%$ y $18,4 \%$ para la articulación derecha e izquierda, valores similares a los encontrados por Manfredini y col. (8) con una frecuencia del $20 \%$ para las articulaciones.

En particular, se puede observar un alto porcentaje de pacientes con afecciones inflamatorias y degenerativas de las articulaciones y; el más bajo porcentaje de $7.9 \%$ de pacientes solo con diagnóstico de trastornos musculares, lo cual es cercano al 4,5\% reportado en el estudio de Manfredini y col. (8).
La baja frecuencia de diagnósticos de dolor miofascial es particularmente confirmado por Manfredini y col. (24) y Yap y col. (26), aunque se reportó una prevalencia más alta de trastorno muscular con respecto a la presente investigación.

Manfredini y col. (8) proponen la hipótesis, que la prevalencia de trastornos musculares ha sido sobreestimada, ya que se confunde el diagnóstico de hiperalgesia muscular con supuesto diagnóstico de dolor miofascial(8).

En relación al eje II, la frecuencia de los aspectos psicosociales según género se evidenció en mayor proporción en mujeres con $100 \%$, similar con otros estudios $(4,25)$.

El dolor crónico se presentó en el 43,4\%, dentro de éstos, el grado I fue el más frecuente (28,9\%). Estos resultados son similares a los encontrados por Flores (6) el año 2008.

Los datos reportados en la presente investigación no coinciden con el estudio de Manfredini y col. (8) probablemente por la gran diferencia en el tamaño muestral (4).

La depresión se presentó en $92,1 \%$, lo cual fue muy alto en comparación a otros estudios que encontraron resultados de $39 \%$ a $65 \%$, lo que se puede explicar por el alto nivel de estrés al que estuvo sometida la muestra. Sin embargo, estos resultados no indican un diagnóstico psiquiátrico formal.

La somatización se presentó en el 92,1\%, cercano a lo referido en la literatura que muestra un porcentaje de $45 \%$ a $85 \%$ (26). Al respecto, Hirsch y col. (25) mencionando el estudio de LeResche, señalan que síntomas de somatización así como quejas de dolor fuera de la región facial son factores de riesgo para desarrollar TTM. Teniendo en cuenta que en el presente estudio toda la muestra presentó TTM, el porcentaje de somatización también es elevado.

Finalmente, al relacionar los diagnósticos del eje I con los valores psicosociales del eje II, los resultados son coincidentes con otros estudios $(3,21)$, observándose una correlación positiva entre trastornos musculares y grado de dolor crónico. 
En conclusión, la relación entre diagnóstico y aspecto psicosocial de los TTM se presentó en los trastornos musculares y grado de dolor crónico, lo que nos indica que a medida que aumenta la severidad de trastorno muscular existe un mayor grado de dolor crónico. El desplazamiento del disco articular con reducción fue más frecuente para los TTM, con predominio en mujeres.

\section{Correspondencia:}

\section{Felipe Enrique Lozano Castro}

Av. Arequipa 1851, Of. 309 Lince. Lima, Perú.

Correo electrónico: felipelozanoc@gmail.com

\section{REFERENCIAS BIBLIOGRÁFICAS}

1. Medina A. Prevalencia de trastornos temporomandibualres y su relación con la pérdida de soporte oclusal posterior en adultos. [Tesis Bachiller]. Lima: Universidad Nacional Mayor de San Marcos; 2010.

2. Nishiyama A, Kino K, Sugisaki M, Tsukagoshi K. A survey of influence of work environment on temporomandibular disorders-related symptoms in Japan. Head \& Face Medicine. 2012; 8(1): 24.

3. Manfredini D, Ahlberg J, Winocur E, et al. Correlation of RDC/TMD axis diagnoses and axis II pain-related disability. A multicenterstudy. Clin Oral Invest. 2011; 15 (5): 749-756.

4. Manfredini D, Winocur E, Ahlberg J, GuardaNardini L, Lobbezoo F. Psychosocial impairment in temporomandibular disordes patients. RDC/TMD axis II findings from a multicentre study. J Dent. 2010; 38(10): 765-772.

5. Dworkin S. Perspectives on the interaction of biological, psychological, and social factors in TMD. J Am Dent Assoc 1994; 125 (7): 856-63.

6. Flores M. Estudio comparativo del índice de criterios diagnósticos de los trastornos temporomandibulares y el índice de Helkimo en una población de estudiantes de odontología en Sinaloa México. [Tesis Doctoral]. México. Universidad de Granada, 2008.

7. Fleitas A, Arellano L, Terán A. Determinación de signos y síntomas de trastornos temporomandibulares en pacientes adultos de odontología de la Universidad de los Andes. Rev Od Los Andes. 2010; 5(2): 14-24.

8. Manfredini D, Piccotti F, Ferronato G, Guarda-Nardini L. Age peaks of different RDC/TMD diagnoses in a patient population. J Dent. 2010; 38 (5): 392-399.

9. Hormiga C, Bonet M, Alodia C, Jaimes A. Prevalencia de síntomas y signos de trastornos temporomandibulares en una población universitaria del área metropolitana de Bucaramanga, Santander. U Científico. 2009;
$1(14) 80-91$.

10. Jiménez Z, De los Santos L, Sáez R, García I. Prevalencia de los trastornos temporomandibulares en la población de 15 años y más de la Ciudad de La Habana. Rev Cubana Estomatol. 2007 ; 44(3): 10-12.

11. Al-Jabrah O, Al-Shumailan Y. Prevalence of temporomandibular disorder signs in patients with complete versus partial dentures. Clin Oral Invest. 2006; 10 (3): 167-173.

12. Vojdani M, Bahrani F, Ghadiri P. The study of relationship between reported temporomandibular symptoms and clinical dysfunction index among university students in Shiraz. J Dent Res.2012; 9(2): 221-5.

13. Bonjardim L, Lopes-Filho R, Amado G, Albuquerque $\mathrm{R}$, Goncalves S. Association between symptoms of temporomandibular disorders and gender, morphological occlusion, and psychological factors in a group of university students. Indian J Dent Res. 2009; 20(2): 190-194.

14. Corsini G, Fuentes R, Bustos L, et al. Determinación de los signos y síntomas de los trastornos temporomandibulares, en estudiantes de 13 a 18 años de un colegio de la comuna de Temuco, Chile. Int J.Morphol. 2005; 23(4):345-352.

15. Rigoldi L, Duarte M, Pereira L, Midori P, Rodrigues R. Signs and symptoms of temporomandibular disorders in adolescents. Braz Oral Res. 2005; 19(2): 93-98.

16. Dworkin S, LeResche L. The Research Diagnostic Criteria for Temporomandibular Disorders (RDC/ TMD). J Orof Painl.1992; 12(36): 669-672.

17. Zielinsky L. Batería de nueve tests de Krogh - Paulsen para determinar la existencia de disfunción del sistema estomatognático. Ateneo Arg. de Odontología. 1982; 12 (1): 28-29.

18. Cornejo S J. Sensibilidad y especificidad del Indice de Krogh-Paulsen en el diagnóstico de los trastornos temporomandibulares. Odontología San Marquina. 1999; 1(3): 16-20.

19. La O Salas N, Corona M, Rey B, Arias Z, Perdomo $X$. Gravedad de la disfunción temporomandibular. MEDISAN. 2006; 10(2): 0-0

20. Marroquín RR. Confiabilidad y validez de los instrumentos de validación. [revista en internet] 2012 septiembre-diciembre. [acceso 14 de Junio 2013]. Disponible en: http//www.une.edu.pe/./ SESION4/Confiabilidad $\% 20 \mathrm{y} \% 20$ Validez $\% 20 \mathrm{de} \% 2$

21. Schulz R, Moya M, Reuss P, Ivanovic S, Díaz M. Relación entre los diagnósticos del eje I (físico) y el eje II (psicosocial), según los Criterios Diagnósticos para la Investigación de Trastornos Temporomandibulares (RDC/TMD), en una población chilena. Revista Dental de Chile. 2011; 102(3): 24-29.

22. Quiroz K. Prevalencia de tratsornos 
temporomandibulares en pacientes de 12 a 17 años con depresión atendidos en el servicio de psiquiatria del Hospital Nacional Guillermo Almenara Irigoyen durante el periodo mayo-setiembre del 2010. [Tesis Bachiller] Lima.. Universidad Inca Garcilaso de la Vega, 2010. .

23. Espinosa I, Lara C, Lara A, Saavedra M, y Vargas H. Comparación de los aspectos psicosociales (eje II) de los pacientes con trastornos temporomandibulares, de acuerdo a la combinación de diagnósticos físicos (eje I) de los criterios diagnósticos para la investigación de los trastornos temporomandibulares (CDI/TTM). Rev O. 2009; 10(30): 477-481.
24. Hernández S R, Fernández CC, Baptista LP. Metodología de la Investigación. 4th ed. México: Mc Graw Hill; 2006.

25. Hirsch C, Turp J. Temporomandibular pain and depression in adolescents-a case- control study. Clin Oral Invest. 2010; 14(2): 145-151. Yap A, Tan KBC, Chuan K, Tan H. Depression and somatisation in patients with temporomandibular disorders. Journal of Prosthetic Dentistry. 2002; 88(5): 479-484.

Recibido: 05/02/2014

Aceptado: 10/04/2014 